\title{
Hypotensive Activity of Moringa oleifera Lam (Moringaceae) Root Extracts and its Volatile Constituents
}

\author{
Aisha Sana ${ }^{1 *}$, Rubeena Saleem ${ }^{1,2}$ and Shaheen Faizi ${ }^{3}$ \\ ${ }^{1}$ Department of Pharmaceutical Chemistry, Faculty of Pharmacy, ${ }^{2}$ Dr. HMI Institute of Pharmacology \& Herbal Sciences, \\ Hamdard University, Karachi-74600, ${ }^{3} \mathrm{HEJ}$ Research Institute of Chemistry, International Center for Chemical and Biological \\ Sciences, University of Karachi, Karachi-75270, Pakistan
}

*For correspondence: Email: aishasana80@gmail.com

Received: 26 January 2015

Revised accepted: 24 April 2015

\begin{abstract}
Purpose: To explore the hypotensive activity and chemical composition of Moringa oleifera Lam (Moringaceae) roots.

Methods: The fresh roots of $M$. oleifera was cut into small pieces and successively extracted with petroleum ether (PE) and dichloromethane (DC). PE extract was further divided into MRP and MRP -1 . $D C$ extract showed a thick mass during evaporation which was separated as MRDC - IN. The mother liquor left was divided into MRDC and MRDC -1. All residues were analyzed by gas chromatographymass spectroscopy (GC-MS) using ZB-5 column. Identification of each extract and fraction was based on comparison of their retention indices (RI), by co-injecting authentic compounds, as well as by comparing literature data available in NIST Standard Reference Database. Hypotensive activity was determined on urethane-anesthetized normotensive Sprague Dawly rats.

Results: Petroleum ether (MRP) and dichloromethane (MRDC) extracts of $M$. oleifera roots showed $50.06 \pm 3.48$ and $48.16 \pm 1.79 \%$ fall in mean arterial blood pressure (MABP), respectively, at a dose of $30 \mathrm{mg} / \mathrm{kg}$ ( $p<0.01$ and $p<0.05$, respectively) compared with control. GC-MS analysis of MRP and MRDC extracts and fractions resulted in the identification of seventy four (74) compounds. Methyl hexadecanoate $(\mathbf{7}, 20.3 \%)$, stigmastan - 3, 5, diene (24, $19.32 \%)$, methyl 14-hydroxy-5tetradecenoate (9,19.22 \%), 1,11 diphenyl undecane (47, $18.78 \%)$ and cyclopentanyl hexadecane (39, $14.44 \%)$ were the major constituents among the various hydrocarbons, fatty acids, esters, alcohols, aldehydes, isothiocyanate, aromatics, steroids, terphenyl and sulphur-containing compounds. Conclusion: The findings reveal the hypotensive potential of $M$. oleifera roots and the presence of specific hydrocarbons, fatty acid esters, thioureides, steroids and isothiocyanates in active fractions. Further study is required to determine the suitability of the plant as an antihypertensive remedy.
\end{abstract}

Keywords: Moringa oleifera, Methyl hexadecanoate, Methyl 14-hydroxy-5-tetradecenoate, Petroleum ether, Stigmastan - 3, 5, diene, Cyclopentanyl hexadecane

Tropical Journal of Pharmaceutical Research is indexed by Science Citation Index (SciSearch), Scopus, International Pharmaceutical Abstract, Chemical Abstracts, Embase, Index Copernicus, EBSCO, African Index Medicus, JournalSeek, Journal Citation Reports/Science Edition, Directory of Open Access Journals (DOAJ), African Journal Online, Bioline International, Open-J-Gate and Pharmacy Abstracts

\section{INTRODUCTION}

Moringa oleifera, Lam is one of the best known and widely utilized specie among the thirteen known species of the monogeneric family Moringaceae. It is native to Pakistan and India, and widely cultivated throughout the world $[1,2]$.
Due to its implausible dietetic and therapeutical values, it is known as a "Miracle tree" and has been declared as complete and natural nutrition for the tropics and famine hit areas [3]. It is a prolific producer of diverse secondary metabolites including glycosides preferentially rhamnosides of carbamates, thiocarbamates, 
nitriles, benzyl glucosinolates, thiocyanates, isothiocyanates and oxazolidine-2-thiones. Pharmacological screening of its leaf extracts exhibited hypotensive [4], cardioprotective [5], hepatoprotective [6], antidiabetic [7] and antiulcerogenic activities [8]. The pods are hypotensive [9] while seeds showed antibacterial [10], antitumor [11], anti-inflammatory and antispasmodic activities [12]. Roots were able to depress the central nervous system by producing analgesia and potentiate the analgesic effect of morphine [13]. Aurantiamide acetate and 1,3 dibenzyl urea isolated from roots showed significant anti inflammatory, antiarthritic and analgesic activity mediated through TNF- alpha, interleukin-2 and cytokines inhibition [14].

In recent decades therapeutic preparations like Septilin for respiratory tract infection, Rumalaya for arthralgia and Pro-lacta of $M$. oleifera have been marketed [15]. This paper reports the hypotensive evaluation and GC-MS analysis of $M$. oleifera roots constituents detected in winter, which are markedly different from those analyzed during summer [16]. Earlier volatile constituents from leaves [17], flowers [18] and pods [19] of $M$. oleifera have already been reported in literature.

\section{EXPERIMENTAL}

\section{Plant material}

Fresh roots of $M$. oleifera $(10 \mathrm{~kg})$ were collected in November 2007 from HEJ-ICCBS Garden University of Karachi, Karachi Pakistan. A voucher specimen (no. $66250 \mathrm{KUH}$ ) was deposited in the herbarium of Department of Botany, University of Karachi, where it was authenticated by Mr Abrar Hussain.

\section{Extraction}

Fresh roots of $M$. oleifera was cut into small pieces (1 - 2 inch) and successively extracted with petroleum ether (PE) and dichloromethane (DC) at room temperature for three days. PE extract was divided into two layers during evaporation. Both layers were separated and evaporated to residual masses MRP (upper layer, $3.14 \mathrm{~g}$ ) and MRP - 1 (lower layer, $0.27 \mathrm{~g}$ ). DC extract showed a thick mass settled at the bottom of round bottom flask during evaporation on rotavapour. It was separated, dried and weighed as MRDC - IN (2.25 g). Mother liquor left was further evaporated which when concentrated divided into two layers. Both layers were separated and evaporated to thick residues. Upper layer furnished MRDC (4.17 g) while lower layer gave MRDC - 1 (8.89 g).

\section{Gas chromatography mass spectrometry}

For GC-MS 6890 N Agilent gas chromatograph coupled with a JMS $600 \mathrm{H}$ JEOL mass spectrometer was used. The compound mixture was separated on a fused silica capillary ZB-5 column, $(30 \mathrm{~m} \times 0.32 \mathrm{~mm}) 0.22 \mu \mathrm{m}$ film thickness in a temperature program from 50 to $260{ }^{\circ} \mathrm{C}$ with a rate of $4{ }^{\circ} \mathrm{C} \mathrm{min}{ }^{-1}$ with $3 \mathrm{~min}$ hold. The injector was set at $240{ }^{\circ} \mathrm{C}$ and the flow rate of helium carrier gas was $1 \mathrm{ml} \mathrm{min}^{-1}$. The El mode JMS $600 \mathrm{H}$ JEOL mass spectrometer had ionization volt $70 \mathrm{eV}$, electron emission $100{ }^{\circ} \mathrm{A}$. Sample was injected manually in split mode. Ratio of sample in split mode was 1:50. Identification of each extract and fractions were based on comparison of their retention indices (RI) calculated according to the Kovats formula, using n-alkanes (C9 - C33) (sigma -Aldrich, Germany) as standards under the same chromatographic conditions and in some cases by co-injection with authentic compounds as well as by following the characteristic mass fragmentation patterns of known compounds. Retention Indices were also compared with literature data available in National Institute of Standards and Technology Standard Reference Database. The relative percentage amount of each component was calculated by comparing its average peak area to the total areas.

\section{Animals}

Normotensive Sprague Dawley rats (both sex, $220-250 \mathrm{~g}$ ) were housed in the animal house of Dr. HMI Institute of Pharmacology and Herbal Sciences in appropriate cages at $21-23{ }^{\circ} \mathrm{C}$. They were maintained at $12 \mathrm{~h}$ of an alternate light and dark cycle with free access to water and standard diet ad libitum. The maintenance and handling of laboratory animals and the experiments conducted follow the protocols based on internationally accepted standard guidelines of the Institutional Ethical Committee. The experimental procedures were performed according to international guidelines [20] and approved by the instutional ethical committee for handling laboratory animals (Ref no. HU/Dr.HMIIPHS/2013/11).

\section{Hypotensive activity}

Rats were anesthetized with urethane $(1.2 \mathrm{gm} /$ $\mathrm{kg}$ i.p.). The trachea was exposed and cannulated with a polyethylene cannula to facilitate spontaneous respiration. Drugs were injected $(0.2-0.25 \mathrm{ml})$ through a polyethylene cannula inserted into the extrajuglar vein followed by a saline flush $(0.2 \mathrm{ml})$. The arterial blood pressure was recorded from the carotid 
artery via an arterial cannula connected to research grade blood pressure transducer (Harvard, 60-3003) coupled with four channel Harvard oscillograph (Curvilinear, 50-9307)(UK). The temperature of the animals was maintained at $37^{\circ} \mathrm{C}$ by over head heating lamp. The mean blood pressure was calculated as the sum of the diastolic blood pressure plus one-third pulse width. Changes in blood pressure were expressed as the percent of control obtained immediately before the administration of test substance. Acetylcholine (Ach) (Merck) at a dose of $1 \mu \mathrm{g} / \mathrm{kg}$ was used as positive control and atropine sulphate $(0.1 \mathrm{mg} / \mathrm{kg})(\mathrm{C}$. H. Beohringer Sohn Ingelheim Rhein, Germany) as muscarinic antagonist. MRP, MRP - 1 and MRDC were soluble in $5 \%$ Tween 80 and others in normal saline.

\section{Statistical analysis}

Changes in blood pressure were compared using Students t-test (IBM SPSS Statistics for Windows, Version 19.0. Armonk, NY 2010). $P<$ 0.05 was considered to be significant.

\section{RESULTS}

GC-MS (vide Tables 1-2) of MRP showed the presence of twenty nine compounds including five aromatics $(1-4,14)$, two diterpenes $(11,12)$, an isothiocyanate (21), a steroid (24) and six thioureido polymers $(13,15,17,19,22,23)$. MRP-1 indicated eight compounds specifically isocyanate (3), isothiocyanate (21), thioureido (23), pyridine (27) and sesquiterpene (28). Analysis of MRDC - IN revealed eleven constituents, particularly long chain aromatic isothiocyanate (21), an aromatic urea (30) and three steroids (24, 31 and 32). MRDC (Tables 14 ) showed the presence of eighteen compounds out of which six $(33,36,40,45,46$ and 47) contained both aromatic and aliphatic moieties. GC-MS study of MRDC - 1 detected thirty eight compounds together with fourteen aromatics (3, $10,33,46,48$ - 53, 58, 65, 67, 71), one lactone (56), one ketone (62) and four aldehydes $(63,66$, $68,70)$.

Intravenous administration of extracts in anesthetized rats showed changes in systolic, diastolic and mean arterial blood pressure (MABP). Intravenous injection of positive control Ach at the dose of $(1 \mu \mathrm{g} / \mathrm{kg})$ showed $(30.63 \pm 3.5$ $\%)$ fall in MABP while that of normal saline (0.9 $\% \mathrm{NaCl}$ ) was insignificant. Hypotensive evaluation of MRP showed significant fall in
MABP at $3 \mathrm{mg} / \mathrm{kg}(41.84 \pm 4.74 \%, p<0.01)$ and $30 \mathrm{mg} / \mathrm{kg}(50.06 \pm 3.48 \%, p<0.01)$.

Hypotensive effect was comparable at both doses however; duration of activity was increased with dose increments (60 sec and 120 $\mathrm{sec}$ ) at 3 and $30 \mathrm{mg} / \mathrm{kg}$ respectively. MRP - 1 displayed dose dependent hypotensive effect at $3 \mathrm{mg} / \mathrm{kg}(26.2 \pm 5.06 \%, p<0.05)$ and $30 \mathrm{mg} / \mathrm{kg}$ (42.58 $\pm 1.21, p<0.05)$ with same duration (41 s) at both doses. MRDC - IN and MRDC - 1 displayed significant $(p<0.05)$ dose-dependant fall in MABP $(28.05 \pm 3.04,49.78 \pm 3.83$ and $22.51 \pm 1.893,38.57 \pm 0.27 \%$, respectively at 3 and $30 \mathrm{mg} / \mathrm{kg}$ ). Duration of action increased up to $25 \mathrm{~min}(1500 \mathrm{~s})$ in case of MRDC -IN at higher dose while other doses of MRDC - IN and MRDC - 1 remained effective for about $34 \mathrm{~s}$.

MRDC showed comparable behavior (46.61 \pm 1.78 and $48.16 \pm 1.79 \%, p<0.05)$ at both doses while duration of action was increased at 30 $\mathrm{mg} / \mathrm{kg}(95.66 \mathrm{sec}$ ) as compared to $3 \mathrm{mg} / \mathrm{kg}$ (34 $\mathrm{sec})$. MRP and MRP - 1 did not show any change in MABP when administered in rats pretreated with atropine sulphate, however, MRDC - IN, MRDC - 1 and MRDC remained impassive by atropine blockade.

\section{DISCUSSION}

GC-MS analysis of extracts and fractions culminated in the detection of eighty five compounds out of which seventy four have been identified. Not unexpectedly, hydrocarbons, and fatty acid esters were found as commonly occurring constituents. Hydrocarbons range from C17 to C33 as di, mono and unsubstituted compounds. 1, 11 diphenyl undecane (47, 18.78 $\%)$ and cyclopentanyl hexadecane $(39,14.44 \%)$ were major hydrocarbon of MRDC. Among fatty acid esters, methyl esters dominate and ranges from tetradecanoate to tetracosanoate. Methyl hexadecanoate $(7,20.3 \%)$ in MRDC and methyl 14-hydroxy-5-tetradecenoate $(\mathbf{9}, 19.22 \%)$ in MRP - 1 were main esters. Isothiocyanates have been detected in all extracts except MRDC while halogenated derivatives were identified only in MRDC and MRDC - 1 .

The characteristic feature of MRP is the presence of new and unique thioureido polymers $(14,15,17,19,22,23)$ that make approximately $19 \%$ of extract. They exhibited several mass fragments with characteristic difference of 74 amu indicating the possible loss of (-NH-CS-NH) ${ }^{+}$ from corresponding molecular fragments/ molecular ion peak. Steroids (approximately 37 
Table 1: Volatile constituents (1-27) of various extracts of Moringa oleifera roots

\begin{tabular}{|c|c|c|c|c|c|c|c|}
\hline$\overline{\mathbf{S} / \mathbf{N}}$ & Compounds $^{\text {g }}$ & $\begin{array}{l}\text { Mol. formula } \\
\text { (M.w) }\end{array}$ & $\mathbf{R I}^{\mathbf{c}}$ & $\mathbf{R I}^{\prime}$ & $\begin{array}{c}\text { Basis of } \\
\text { identification }\end{array}$ & Extract & $\begin{array}{l}\text { Content } \\
(\%)\end{array}$ \\
\hline 1 & Benzaldehylde $^{c}(1)$ & $\begin{array}{l}\mathrm{C}_{7} \mathrm{H}_{6} \mathrm{O} \\
(106)\end{array}$ & 958 & 961 & MS,RI & MRP & 0.17 \\
\hline 2 & 2-Amyl furan ${ }^{\mathrm{c}}(2)$ & $\begin{array}{c}\mathrm{C}_{9} \mathrm{H}_{14} \mathrm{O} \\
(138)\end{array}$ & 987 & 1001 & MS,RI & MRP & 0.32 \\
\hline 3 & $p$-Tolyl isocyanate ${ }^{a, b}(3)$ & $\begin{array}{c}\mathrm{C}_{8} \mathrm{H}_{9} \mathrm{NO} \\
(133)\end{array}$ & 1007 & - & MS & $\begin{array}{l}\text { MRP } \\
\text { MRP-1 }\end{array}$ & $\begin{array}{l}0.78 \\
7.76\end{array}$ \\
\hline & & & & & & MRDC-1 & 0.21 \\
\hline 4 & Benzamide $^{c}(4)$ & $\begin{array}{l}\mathrm{C}_{7} \mathrm{H}_{7} \mathrm{ON} \\
(121)\end{array}$ & 1342 & 1344 & MS,RI & MRP & 0.46 \\
\hline 5 & Octadecane ${ }^{\mathrm{c}}(\mathbf{5})$ & $\begin{array}{c}\mathrm{C}_{18} \mathrm{H}_{38} \\
(254)\end{array}$ & 1800 & 1800 & MS, RI & $\begin{array}{l}\text { MRP } \\
\text { MRDC }\end{array}$ & $\begin{array}{l}0.52 \\
1.87\end{array}$ \\
\hline 6 & Nanodecane ${ }^{\mathrm{b}, \mathrm{c}}(\mathbf{6})$ & $\begin{array}{l}\mathrm{C}_{19} \mathrm{H}_{40} \\
(268)\end{array}$ & 1900 & 1900 & MS, RI & $\begin{array}{c}\text { MRP } \\
\text { MRDC-1 }\end{array}$ & $\begin{array}{l}0.81 \\
0.44\end{array}$ \\
\hline 7 & $\begin{array}{l}\text { Methyl hexadecanoate }{ }^{\mathrm{b}, \mathrm{c}} \\
\text { (7) }\end{array}$ & $\begin{array}{l}\mathrm{C}_{17} \mathrm{H}_{34} \mathrm{O}_{2} \\
(270)\end{array}$ & 1935 & 1933 & MS, RI & $\begin{array}{l}\text { MRP } \\
\text { MRDC - IN } \\
\text { MRDC } \\
\text { MRDC-1 }\end{array}$ & $\begin{array}{l}3.31 \\
3.18 \\
20.3 \\
7.80\end{array}$ \\
\hline 8 & Ethyl hexadecanoate ${ }^{\mathrm{b}, \mathrm{c}}(\mathbf{8})$ & $\begin{array}{l}\mathrm{C}_{18} \mathrm{H}_{30} \mathrm{O}_{2} \\
(284)\end{array}$ & 2013 & 2026 & MS, RI & $\begin{array}{l}\text { MRP } \\
\text { MRDC } \\
\text { MRDC-1 }\end{array}$ & $\begin{array}{l}2.05 \\
4.86 \\
1.56\end{array}$ \\
\hline 9 & $\begin{array}{l}\text { Methyl 14-hydroxy-5- } \\
\text { tetradecenoate } \mathrm{d}_{\mathrm{d}, \mathrm{e}}(\mathbf{9})\end{array}$ & $\begin{array}{l}\mathrm{C}_{15} \mathrm{H}_{28} \mathrm{O}_{3} \\
\quad(256)\end{array}$ & 2135 & 2130 & RI & $\begin{array}{l}\text { MRP } \\
\text { MRP-1 }\end{array}$ & $\begin{array}{c}8.89 \\
19.22\end{array}$ \\
\hline 10 & $\begin{array}{l}(Z)-11 \text {-Eicosenoic acid }{ }^{\mathrm{b}, \mathrm{c}} \\
(\mathbf{1 0})\end{array}$ & $\begin{array}{c}\mathrm{C}_{20} \mathrm{H}_{38} \mathrm{O}_{2} \\
(310)\end{array}$ & 2286 & 2300 & MS, RI & $\begin{array}{c}\text { MRP } \\
\text { MRDC-1 }\end{array}$ & $\begin{array}{l}3.01 \\
2.09\end{array}$ \\
\hline 11 & $\begin{array}{l}\text { Abieta-9(11),8(14),12-trien- } \\
\text { 12-ol (trans) }{ }^{\text {d.e }}(\mathbf{1 1})\end{array}$ & $\begin{array}{c}\mathrm{C}_{20} \mathrm{H}_{30} \mathrm{O} \\
(286)\end{array}$ & 2324 & 2325 & MS, RI & MRP & 3.48 \\
\hline 12 & $\begin{array}{l}\text { Abieta-8,11,13-trien-7- } \\
\text { one }^{\text {d.e }}(\mathbf{1 2})\end{array}$ & $\begin{array}{c}\mathrm{C}_{20} \mathrm{H}_{28} \mathrm{O} \\
(284)\end{array}$ & 2341 & 2315 & MS, RI & MRP & 3.07 \\
\hline 13 & $\begin{array}{l}\text { Hepta thioureido- bis }-1,1^{\prime}- \\
\text { methylene amine }{ }^{\mathrm{e}}(\mathbf{1 3})\end{array}$ & $\begin{array}{c}\mathrm{C}_{9} \mathrm{H}_{22} \mathrm{~N}_{16} \mathrm{~S}_{7} \\
(578)\end{array}$ & 2370 & - & MS & MRP & 3.56 \\
\hline 14 & $\begin{array}{l}\text { Ethene } 1,1^{\prime}-\text { bis- } p \text {-toulenyl } \\
\text { sulfide }^{\mathrm{e}}(\mathbf{1 4})\end{array}$ & $\begin{array}{l}\mathrm{C}_{16} \mathrm{H}_{16} \mathrm{~S}_{2} \\
(272)\end{array}$ & 2414 & - & MS & $\begin{array}{c}\text { MRP } \\
\text { MRDC-1 }\end{array}$ & $\begin{array}{l}2.90 \\
1.54\end{array}$ \\
\hline 15 & $\begin{array}{l}\text { Octa thioureido- bis-1,1'- } \\
\text { methylene amine }{ }^{\mathrm{e}}(\mathbf{1 5})\end{array}$ & $\begin{array}{c}\mathrm{C}_{10} \mathrm{H}_{24} \mathrm{~N}_{18} \mathrm{~S}_{8} \\
(652)\end{array}$ & 2517 & - & MS & MRP & 4.05 \\
\hline 16 & Unidentified & - & 2523 & & - & MRP & 2.78 \\
\hline 17 & $\begin{array}{l}\text { 3,7-Dimethyl pentacosane } \\
\text { d,e (16) }\end{array}$ & $\begin{array}{l}\mathrm{C}_{27} \mathrm{H}_{56} \\
(380)\end{array}$ & 2612 & 2608 & MS,RI & MRP & 1.77 \\
\hline 18 & $\begin{array}{l}\text { Octa thioureido- bis }-1,1^{\prime}- \\
\text { methane }^{e}(17)\end{array}$ & $\begin{array}{c}\mathrm{C}_{10} \mathrm{H}_{22} \mathrm{~N}_{16} \mathrm{~S}_{8} \\
(622)\end{array}$ & 2667 & - & MS & MRP & 2.03 \\
\hline 19 & 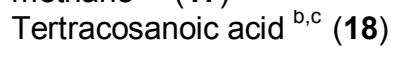 & $\begin{array}{c}\mathrm{C}_{24} \mathrm{H}_{48} \mathrm{O}_{2} \\
(368)\end{array}$ & 2725 & 2685 & MS,RI & MRP & 2.45 \\
\hline 20 & $\begin{array}{l}\text { Hepta thioureido-bis-1,1'- } \\
\text { thioamide }{ }^{\mathrm{e}}(\mathbf{1 9})\end{array}$ & $\begin{array}{c}\mathrm{C}_{9} \mathrm{H}_{18} \mathrm{~N}_{16} \mathrm{~S}_{9} \\
(638)\end{array}$ & 2778 & - & MS & MRP & 2.88 \\
\hline 21 & $\begin{array}{l}\text { 3-Methyl heptacosane } e^{\mathrm{a}, \mathrm{e}} \\
(\mathbf{2 0})\end{array}$ & $\begin{array}{c}\mathrm{C}_{28} \mathrm{H}_{58} \\
(394)\end{array}$ & 2764 & 2773 & MS,RI & MRP & 1.92 \\
\hline 22 & $\begin{array}{l}\text { 15- Phenyl pentadecanyl } \\
\text { isothiocyanate }{ }^{\mathrm{e}}(\mathbf{2 1})\end{array}$ & $\begin{array}{c}\mathrm{C}_{22} \mathrm{H}_{35} \mathrm{NS} \\
(345)\end{array}$ & 2930 & - & - & $\begin{array}{c}\text { MRP } \\
\text { MRP-1 } \\
\text { MRDC - IN }\end{array}$ & $\begin{array}{l}4.10 \\
9.40 \\
6.36\end{array}$ \\
\hline 23 & $\begin{array}{l}\text { Octa thioureido 1-hydroxy- } \\
1,1^{\prime} \text { amino methylene }{ }^{\mathrm{e}} \\
(\mathbf{2 2})\end{array}$ & $\begin{array}{c}\mathrm{C}_{10} \mathrm{H}_{24} \mathrm{~N}_{18} \mathrm{~S}_{8} \mathrm{O} \\
(668)\end{array}$ & 3017 & - & MS & MRP & 2.89 \\
\hline 24 & Unidentified & - & 3020 & - & - & MRP & 2.46 \\
\hline
\end{tabular}

${ }^{a}$ Mass spectra were compared with literature fragmentation pattern given in National Institute of Standards and Technology Standard Reference Database Number 69; ${ }^{b}$ Mass and Retention index comparable with standard compound injected under similar condition; ${ }^{C}$ Mass and Retention index comparable with values given in literature available in NIST database; ${ }^{d}$ Retention index match with literature available in NIST database; ${ }^{e}$ Compound tentatively identified according to observed mass fragmentation pattern; ${ }^{f}$ Retention index value match with non equivalent column; ${ }^{g}$ order of elution is given on column (ZB-5) 
Table 2: Volatile constituents (25-46) of various extracts of Moringa oleifera roots

\begin{tabular}{|c|c|c|c|c|c|c|c|}
\hline$\overline{\mathrm{S} / \mathrm{N}}$ & Compounds $^{g}$ & $\begin{array}{l}\text { Mol. formula } \\
\text { (M.w) }\end{array}$ & $\mathbf{R I}^{\mathrm{c}}$ & $\mathbf{R I}^{\prime}$ & $\begin{array}{c}\text { Basis of } \\
\text { identification }\end{array}$ & Extract & $\begin{array}{c}\text { Content } \\
(\%)\end{array}$ \\
\hline 25 & Unidentified & - & 3047 & - & - & MRP & 2.47 \\
\hline 26 & $\begin{array}{l}\text { Benzyl hexathioureido } \\
\text { propane }^{\mathrm{e}}(\mathbf{2 3})\end{array}$ & $\begin{array}{c}\mathrm{C}_{16} \mathrm{H}_{26} \mathrm{~N}_{12} \mathrm{~S}_{6} \\
(578)\end{array}$ & 3070 & - & MS & $\begin{array}{l}\text { MRP } \\
\text { MRP-1 }\end{array}$ & $\begin{array}{l}3.19 \\
7.14\end{array}$ \\
\hline 27 & $\begin{array}{l}\text { Stigmastan-3,5-diene } e^{\mathrm{d}, \mathrm{e}} \\
\text { (24) }\end{array}$ & $\begin{array}{l}\mathrm{C}_{29} \mathrm{H}_{48} \\
(396)\end{array}$ & 3036 & 3040 & MS, RI & $\begin{array}{c}\text { MRP } \\
\text { MRDC-IN }\end{array}$ & $\begin{array}{l}5.66 \\
19.32\end{array}$ \\
\hline 28 & $\begin{array}{l}\text { 3,7-Dimethyl triacontane } \\
\mathrm{d}, \mathrm{e} \\
(\mathbf{2 5})\end{array}$ & $\begin{array}{l}\mathrm{C}_{32} \mathrm{H}_{66} \\
(450)\end{array}$ & 3113 & 3110 & MS, RI & MRP & 1.14 \\
\hline 29 & $\begin{array}{l}\text { 9,13-Dimethyl } \\
\text { hentriacontane }^{\mathrm{d}, \mathrm{e}}(\mathbf{2 6})\end{array}$ & $\begin{array}{l}\mathrm{C}_{33} \mathrm{H}_{68} \\
(464)\end{array}$ & 3161 & 3162 & MS, RI & MRP & 1.21 \\
\hline 30 & 2,5 Diethyl pyridine ${ }^{\mathrm{c}}(\mathbf{2 7})$ & $\begin{array}{c}\mathrm{C}_{9} \mathrm{H}_{13} \mathrm{~N} \\
(135)\end{array}$ & 1393 & 1422 & MS, RI & MRP-1 & 2.42 \\
\hline 31 & $\begin{array}{l}\text { 1- } \beta \text {-Acetoxy furano-3- } \\
\text { eudesmene }^{\mathrm{d}, \mathrm{e}}(\mathbf{2 8})\end{array}$ & $\begin{array}{l}\mathrm{C}_{17} \mathrm{H}_{24} \mathrm{O}_{3} \\
\quad(276)\end{array}$ & 1978 & 1978 & MS, RI & MRP-1 & 4.80 \\
\hline 32 & $\begin{array}{l}\text { 1,10 Diphenyl decane } \\
\text { (29) }\end{array}$ & $\begin{array}{l}\mathrm{C}_{22} \mathrm{H}_{30} \\
(294)\end{array}$ & 2581 & - & MS & MRP-1 & 8.62 \\
\hline 33 & Unidentified & - & 3058 & - & - & MRP-1 & 6.94 \\
\hline 34 & Unidentified & - & 2165 & - & - & MRDC-IN & 2.65 \\
\hline 35 & Unidentified & - & 2427 & - & - & MRDC-IN & 1.88 \\
\hline 36 & Unidentified & - & 2678 & - & - & MRDC-IN & 22.27 \\
\hline 37 & $\begin{array}{l}\text { N,N- Dibenzyl undecanyl } \\
\text { urea }^{\mathrm{e}}(\mathbf{3 0})\end{array}$ & $\begin{array}{l}\mathrm{C}_{26} \mathrm{H}_{38} \mathrm{~N}_{2} \mathrm{O} \\
\quad(394)\end{array}$ & 2930 & - & MS & MRDC-IN & 4.58 \\
\hline 38 & $\begin{array}{l}\text { Ergosta-5,22-dien-3-ol, } \\
(3 \beta, 22 \mathrm{E})^{\mathrm{c}}(\mathbf{3 1})\end{array}$ & $\begin{array}{c}\mathrm{C}_{28} \mathrm{H}_{46} \mathrm{O} \\
(398)\end{array}$ & 3017 & 3044 & - & MRDC-IN & 9.36 \\
\hline 39 & Unidentified & - & 3033 & - & - & MRDC-IN & 10.69 \\
\hline 40 & $\begin{array}{l}\text { Stigmastan }-3,5,22 \text { triene }^{\mathrm{e}} \\
(\mathbf{3 2})\end{array}$ & $\begin{array}{l}\mathrm{C}_{29} \mathrm{H}_{46} \\
(394)\end{array}$ & 3044 & 2981 & MS,RI & MRDC-IN & 7.88 \\
\hline 41 & Unidentified & - & 3056 & - & - & MRDC-IN & 7.04 \\
\hline 42 & $\begin{array}{l}\text { o-Amino butyl benzene } \\
\text { (33) }\end{array}$ & $\begin{array}{c}\mathrm{C}_{10} \mathrm{H}_{15} \mathrm{~N} \\
(149)\end{array}$ & 1427 & 1390 & MS, RI & $\begin{array}{l}\text { MRDC } \\
\text { MRDC-1 }\end{array}$ & $\begin{array}{l}2.43 \\
1.59\end{array}$ \\
\hline 43 & $\begin{array}{l}\text { 2-Methyl hexadecane } \\
\text { (34) }\end{array}$ & $\begin{array}{l}\mathrm{C}_{17} \mathrm{H}_{34} \\
(240)\end{array}$ & 1674 & 1666 & MS, RI & MRDC & 0.95 \\
\hline 44 & $\begin{array}{l}\text { Methyl tetradecanoate }{ }^{c} \\
\text { (35) }\end{array}$ & $\begin{array}{l}\mathrm{C}_{15} \mathrm{H}_{30} \mathrm{O}_{2} \\
(242)\end{array}$ & 1708 & 1706 & MS,RI & MRDC & 1.08 \\
\hline 45 & $\begin{array}{l}\text { 1-3 Dibenzyl-3-ethyl } \\
\text { urea }^{\mathrm{e}(36)}\end{array}$ & $\begin{array}{l}\mathrm{C}_{17} \mathrm{H}_{20} \mathrm{~N}_{2} \mathrm{O} \\
\quad(268)\end{array}$ & 1969 & - & MS & MRDC & 2.51 \\
\hline 46 & $\begin{array}{l}\text { Methyl }(E)-9- \\
\text { hexadecenoate }^{\mathrm{c}}(37)\end{array}$ & $\begin{array}{l}\mathrm{C}_{17} \mathrm{H}_{32} \mathrm{O}_{2} \\
(268)\end{array}$ & 1924 & 1912 & MS, RI & MRDC & 2.961 \\
\hline
\end{tabular}

${ }^{a}$ Mass spectra were compared with literature fragmentation pattern given in National Institute of Standards and Technology Standard Reference Database Number 69; ${ }^{b}$ Mass and Retention index comparable with standard compound injected under similar condition; ${ }^{C}$ Mass and Retention index comparable with values given in literature available in NIST database; ${ }^{d}$ Retention index match with literature available in NIST database; ${ }^{e}$ Compound tentatively identified according to observed mass fragmentation pattern; ${ }^{f}$ Retention index value match with non equivalent column; ${ }^{g}$ order of elution is given on column (ZB-5) 
Table 3: Volatile constituents (47-68) of various extracts of Moringa oleifera roots

\begin{tabular}{|c|c|c|c|c|c|c|c|}
\hline $\mathrm{S} / \mathrm{N}$ & Compounds $^{g}$ & $\begin{array}{l}\text { Mol. formula } \\
\text { (M.w) }\end{array}$ & $\overline{\mathbf{R I}^{\mathbf{c}}}$ & $\mathbf{R I}^{\prime}$ & $\begin{array}{c}\text { Basis of } \\
\text { identification }\end{array}$ & Extract & $\begin{array}{c}\text { Content } \\
(\%)\end{array}$ \\
\hline 47 & $\begin{array}{l}\text { (E)-6-Octadecenoic acid } \\
\text { d,e (38) }\end{array}$ & $\begin{array}{l}\mathrm{C}_{18} \mathrm{H}_{34} \mathrm{O}_{2} \\
(282)\end{array}$ & 2085 & 2073 & MS, RI & MRDC & 6.07 \\
\hline 48 & $\begin{array}{l}\text { Cyclopentanyl } \\
\text { hexadecane } \mathrm{d}_{\mathrm{d}, \mathrm{e}}(\mathbf{3 9})\end{array}$ & $\begin{array}{l}\mathrm{C}_{21} \mathrm{H}_{42} \\
(294)\end{array}$ & 2182 & 2167 & $\mathrm{RI}$ & MRDC & 14.44 \\
\hline 49 & $\begin{array}{l}\text { Decyl-3-chlorobenzoate }{ }^{a, e} \\
(\mathbf{4 0 )}\end{array}$ & $\begin{array}{l}\mathrm{C}_{17} \mathrm{H}_{25} \mathrm{ClO}_{2} \\
(296)\end{array}$ & 2189 & 2173 & MS, RI & MRDC & 12.0 \\
\hline 50 & $\begin{array}{l}\text { Methyl (Z)-13- } \\
\text { Octadecenoate }^{\mathrm{d}, \mathrm{e}}(\mathbf{4 1})\end{array}$ & $\begin{array}{l}\mathrm{C}_{19} \mathrm{H}_{36} \mathrm{O}_{2} \\
(296)\end{array}$ & 2151 & 2126 & MS, RI & $\begin{array}{l}\text { MRDC } \\
\text { MRDC-1 }\end{array}$ & $\begin{array}{l}9.02 \\
4.97\end{array}$ \\
\hline 51 & $\begin{array}{l}\text { Propyl heptadecanoate }{ }^{\mathrm{d}, \mathrm{e}} \\
\text { (42) }\end{array}$ & $\begin{array}{c}\mathrm{C}_{20} \mathrm{H}_{40} \mathrm{O}_{2} \\
(312)\end{array}$ & 2221 & 2193 & $\mathrm{RI}$ & MRDC & 4.10 \\
\hline 52 & $\begin{array}{l}\text { (Z)-13-Eicosenoic acid }{ }^{\mathrm{d}, \mathrm{e}} \\
(\mathbf{4 3})\end{array}$ & $\begin{array}{c}\mathrm{C}_{20} \mathrm{H}_{38} \mathrm{O}_{2} \\
(310)\end{array}$ & 2312 & 2365 & MS, RI & MRDC & 7.24 \\
\hline 53 & $\begin{array}{l}\text { 12-Methyl tetracosane }{ }^{\mathrm{d}, \mathrm{e}} \\
(\mathbf{4 4 )}\end{array}$ & $\begin{array}{l}\mathrm{C}_{25} \mathrm{H}_{52} \\
(352)\end{array}$ & 2439 & 2434 & $\mathrm{MS}, \mathrm{RI}$ & MRDC & 5.00 \\
\hline 54 & $\begin{array}{l}\text { N,N-Dibenzyl-3N- pentyl } \\
\text { thiourea }^{\mathrm{e}}(\mathbf{4 5})\end{array}$ & $\begin{array}{c}\mathrm{C}_{20} \mathrm{H}_{26} \mathrm{~N}_{2} \mathrm{~S} \\
(326)\end{array}$ & 2455 & - & MS & MRDC & 5.69 \\
\hline 55 & Dibenzyl phthalate ${ }^{\mathrm{C}}(\mathbf{4 6})$ & $\begin{array}{l}\mathrm{C}_{22} \mathrm{H}_{18} \mathrm{O}_{4} \\
(346)\end{array}$ & 2647 & 2690 & MS,RI & $\begin{array}{l}\text { MRDC } \\
\text { MRDC-1 }\end{array}$ & $\begin{array}{l}5.77 \\
1.37\end{array}$ \\
\hline 56 & $\begin{array}{l}1,11 \text { Diphenyl undecane } \\
\text { (47) }\end{array}$ & $\begin{array}{l}\mathrm{C}_{23} \mathrm{H}_{32} \\
(308)\end{array}$ & 3017 & - & MS & MRDC & 18.78 \\
\hline 57 & $\begin{array}{l}\text { 1-Chloro-2-methyl } \\
\text { benzene }^{c}(\mathbf{4 8})\end{array}$ & $\begin{array}{l}\mathrm{C}_{7} \mathrm{H}_{7} \mathrm{Cl} \\
(126)\end{array}$ & 913 & 955 & $\mathrm{RI}$ & MRDC-1 & 0.06 \\
\hline 58 & Benzyl thiol ${ }^{\mathrm{C}}(49)$ & $\begin{array}{l}\mathrm{C}_{7} \mathrm{H}_{8} \mathrm{~S} \\
(124)\end{array}$ & 969 & 1067 & MS & MRDC-1 & 0.13 \\
\hline 59 & $\begin{array}{l}\text { Benzyl isothiocyanate }{ }^{c} \\
(\mathbf{5 0})\end{array}$ & $\begin{array}{c}\mathrm{C}_{8} \mathrm{H}_{7} \mathrm{NS} \\
(149)\end{array}$ & 1281 & 1320 & $\mathrm{MS}, \mathrm{RI}$ & MRDC-1 & 0.21 \\
\hline 60 & $\begin{array}{l}\text { Methyl 3,3-diphenyl } \\
\text { propanoate }^{\mathrm{e}}(\mathbf{5 1})\end{array}$ & $\begin{array}{l}\mathrm{C}_{16} \mathrm{H}_{16} \mathrm{O}_{2} \\
(240)\end{array}$ & 1692 & - & MS & MRDC- 1 & 0.13 \\
\hline 61 & $\begin{array}{l}4(2 \text { '-Amino) ethyl benzyl } \\
\text { isocyanate }^{\mathrm{e}}(\mathbf{5 2})\end{array}$ & $\begin{array}{c}\mathrm{C}_{10} \mathrm{H}_{12} \mathrm{~N}_{2} \mathrm{O} \\
(176)\end{array}$ & 1781 & - & MS & MRDC-1 & 0.35 \\
\hline 62 & $\begin{array}{l}\text { Di-3-phenyl propyl ether }{ }^{\mathrm{e}} \\
\text { (53) }\end{array}$ & $\begin{array}{c}\mathrm{C}_{18} \mathrm{H}_{22} \mathrm{O} \\
(254)\end{array}$ & 1789 & - & MS & MRDC-1 & 0.48 \\
\hline 63 & Tetradecanoic acid ${ }^{\mathrm{D}, \mathrm{C}}(\mathbf{5 4})$ & $\begin{array}{l}\mathrm{C}_{14} \mathrm{H}_{28} \mathrm{O}_{2} \\
(228)\end{array}$ & 1745 & 1761 & MS, RI & MRDC-1 & 0.27 \\
\hline 64 & $\begin{array}{l}\text { Methyl (Z)-9- } \\
\text { hexadecenoate }^{\mathrm{c}}(\mathbf{5 5})\end{array}$ & $\begin{array}{l}\mathrm{C}_{17} \mathrm{H}_{32} \mathrm{O}_{2} \\
(268)\end{array}$ & 1922 & 1912 & $M S, R I$ & MRDC-1 & 0.95 \\
\hline 65 & $\begin{array}{l}\text { Tetrahydro-6-undecanyl- } \\
2 \mathrm{H}-\text { pyran-2-one }{ }^{\mathrm{c}}(\mathbf{5 6})\end{array}$ & $\begin{array}{c}\mathrm{C}_{16} \mathrm{H}_{30} \mathrm{O}_{2} \\
(254)\end{array}$ & 2070 & 2070 & $M S, R I$ & MRDC-1 & 1.053 \\
\hline 66 & $\begin{array}{l}\text { Methyl heptadecanoate }{ }^{c} \\
(\mathbf{5 7})\end{array}$ & $\begin{array}{c}\mathrm{C}_{18} \mathrm{H}_{36} \mathrm{O}_{2} \\
(284)\end{array}$ & 2083 & 2028 & MS, RI & MRDC-1 & 1.04 \\
\hline 67 & $\begin{array}{l}\text { (E)-3,7-Dimethyl octa-2,6- } \\
\text { dienyl-3-chlorobenzoate } \\
\text { (58) }\end{array}$ & $\begin{array}{l}\mathrm{C}_{17} \mathrm{H}_{21} \mathrm{Cl} \mathrm{O}_{2} \\
(292)\end{array}$ & 2176 & 2150 & MS, RI & MRDC-1 & 4.65 \\
\hline 68 & $\begin{array}{l}\text { Methyl (Z)-9- } \\
\text { octadecenoate }^{d}(59)\end{array}$ & $\begin{array}{l}\mathrm{C}_{19} \mathrm{H}_{36} \mathrm{O}_{2} \\
(296)\end{array}$ & 2146 & 2106 & MS, RI & MRDC-1 & 3.47 \\
\hline
\end{tabular}

${ }^{a}$ Mass spectra were compared with literature fragmentation pattern given in National Institute of Standards and Technology Standard Reference Database Number 69; ${ }^{b}$ Mass and Retention index comparable with standard compound injected under similar condition; 'Mass and Retention index comparable with values given in literature available in NIST database; ${ }^{d}$ Retention index match with literature available in NIST database; ${ }^{e}$ Compound tentatively identified according to observed mass fragmentation pattern; ${ }^{f}$ Retention index value match with non equivalent column; ${ }^{g}$ order of elution is given on column (ZB-5) 
Table 4: Volatile constituents (69-85) of various extracts of Moringa oleifera roots

\begin{tabular}{|c|c|c|c|c|c|c|c|}
\hline $\mathbf{S} / \mathbf{N}$ & Compounds $^{g}$ & $\begin{array}{l}\text { Mol. formula } \\
\text { (M.w) }\end{array}$ & $\mathbf{R I}^{\mathbf{c}}$ & $\mathbf{R I}^{\prime}$ & $\begin{array}{c}\text { Basis of } \\
\text { identification }\end{array}$ & Extract & $\begin{array}{c}\text { Content } \\
(\%)\end{array}$ \\
\hline 69 & Methyl octadecanoate ${ }^{d}(60)$ & $\begin{array}{l}\mathrm{C}_{19} \mathrm{H}_{38} \mathrm{O}_{2} \\
(298)\end{array}$ & 2158 & 2139 & MS, RI & MRDC-1 & 1.67 \\
\hline 70 & $\begin{array}{l}\text { Methyl }(Z, Z)-11,14- \\
\text { eicosadienoate }^{\mathrm{d}}(\mathbf{6 1})\end{array}$ & $\begin{array}{l}\mathrm{C}_{21} \mathrm{H}_{38} \mathrm{O}_{2} \\
\quad(322)\end{array}$ & 2250 & 2279 & MS,RI & MRDC-1 & 0.09 \\
\hline 71 & 2-Docosanone ${ }^{\mathrm{c}}(\mathbf{6 2})$ & $\begin{array}{c}\mathrm{C}_{22} \mathrm{H}_{44} \mathrm{O} \\
(324)\end{array}$ & 2408 & 2410 & MS, RI & MRDC-1 & 1.71 \\
\hline 72 & Tricosanal ${ }^{\mathrm{c}}(63)$ & $\begin{array}{c}\mathrm{C}_{23} \mathrm{H}_{46} \mathrm{O} \\
(338)\end{array}$ & 2510 & 2530 & MS, RI & MRDC-1 & 1.73 \\
\hline 73 & $\begin{array}{l}\text { 3,11-Dimethyl pentacosane } \\
d(64)\end{array}$ & $\begin{array}{l}\mathrm{C}_{27} \mathrm{H}_{56} \\
(380)\end{array}$ & 2602 & 2607 & MS, RI & MRDC-1 & 1.87 \\
\hline 74 & $\begin{array}{l}\text { 1-(p-Benzyl) phenyl -7- } \\
\text { phenyl-1-heptene }{ }^{\mathrm{e}}(\mathbf{6 5})\end{array}$ & $\begin{array}{l}\mathrm{C}_{27} \mathrm{H}_{30} \\
(354)\end{array}$ & 2652 & - & MS & MRDC-1 & 1.09 \\
\hline 75 & Pentacosanal ${ }^{\mathrm{c}}(\mathbf{6 6})$ & $\begin{array}{c}\mathrm{C}_{25} \mathrm{H}_{50} \mathrm{O} \\
(366)\end{array}$ & 2719 & 2733 & MS, RI & MRDC-1 & 2.19 \\
\hline 76 & $\begin{array}{l}\text { 1-p-Toluenyl-2-fluoro,4-(3'- } \\
\text { methyl-4' phenyl) phenyl - } \\
5 \text { - methyl benzene e }(\mathbf{6 7})\end{array}$ & $\begin{array}{c}\mathrm{C}_{27} \mathrm{H}_{25} \mathrm{~F} \\
\quad(368)\end{array}$ & 2745 & - & MS & MRDC-1 & 2.23 \\
\hline 77 & Hexacosanal ${ }^{\mathrm{C}}(\mathbf{6 8})$ & $\begin{array}{c}\mathrm{C}_{26} \mathrm{H}_{52} \mathrm{O} \\
(380)\end{array}$ & 2789 & 2830 & MS & MRDC-1 & 2.47 \\
\hline 78 & Methyl tetracosanoate ${ }^{\mathrm{e}}(\mathbf{6 9})$ & $\begin{array}{l}\mathrm{C}_{25} \mathrm{H}_{50} \mathrm{O}_{2} \\
\quad(382)\end{array}$ & 2833 & & MS & MRDC-1 & 1.04 \\
\hline 79 & Heptacosanal ${ }^{\mathrm{c}}(\mathbf{7 0})$ & $\begin{array}{l}\mathrm{C}_{27} \mathrm{H}_{54} \mathrm{O} \\
(394)\end{array}$ & 2918 & 2930 & MS & MRDC-1 & 2.17 \\
\hline 80 & Unidentified & - & 2928 & - & - & MRDC-1 & 3.21 \\
\hline 81 & $\begin{array}{l}\text { 1,4 -Ditoluenyl 2- chloro-5- } \\
\text { methyl -2,5 cyclohexadiene } \\
{ }^{\mathrm{e}}(\mathbf{7 1})\end{array}$ & $\begin{array}{l}\mathrm{C}_{22} \mathrm{H}_{23} \mathrm{Cl} \\
(322)\end{array}$ & 3019 & - & MS & MRDC-1 & 9.09 \\
\hline 82 & $\begin{array}{l}(Z, Z)-9,18- \\
\text { Hentriacontadiene } e^{f}(\mathbf{7 2})\end{array}$ & $\begin{array}{l}\mathrm{C}_{31} \mathrm{H}_{60} \\
(432)\end{array}$ & 3070 & 3055 & $\mathrm{RI}$ & MRDC-1 & 2.24 \\
\hline 83 & 2-Methyl triacontane ${ }^{\dagger}(\mathbf{7 3})$ & $\begin{array}{l}\mathrm{C}_{31} \mathrm{H}_{64} \\
(436)\end{array}$ & 3064 & 3065 & MS, RI & MRDC-1 & 2.06 \\
\hline 84 & $\begin{array}{l}\text { 13-Methyl hentriacontane } \\
\text { d,e (74) }\end{array}$ & $\begin{array}{l}\mathrm{C}_{32} \mathrm{H}_{66} \\
(450)\end{array}$ & 3110 & 3130 & MS, RI & MRDC-1 & 0.78 \\
\hline 85 & Unidentified & - & 3154 & - & - & MRDC-1 & 0.71 \\
\hline
\end{tabular}

${ }^{a}$ Mass spectra were compared with literature fragmentation pattern given in National Institute of Standards and Technology Standard Reference Database Number 69; ${ }^{b}$ Mass and Retention index comparable with standard compound injected under similar condition; 'Mass and Retention index comparable with values given in literature available in NIST database; ${ }^{d}$ Retention index match with literature available in NIST database; ${ }^{e}$ Compound tentatively identified according to observed mass fragmentation pattern; ${ }^{f}$ Retention index value match with non equivalent column; ${ }^{g}$ order of elution is given on column (ZB-5)

$\%$ ) were observed as main class of compounds in MRDC - IN. MRDC - 1 showed presence of most diversified constituents including aldehydes, isocyanates, ketone and lactone along with other mentioned compounds.

All extracts and fractions examined showed significant hypotensive activity, however, as far as mode of action is concerned, PE and DC extracts have opposite behavior. MRP and MRP - 1 mediate through muscarinic receptors as both did not show any change in MABP in atropine pretreated animals. Stimulation of muscarinic receptors by MRP and MRP - 1 may cause the release of nitric oxide or endothelium derived relaxing factors (EDRF) that diffuse in smooth muscle cells and initiate immediate decrease in MABP. MRDC - IN, MRDC - 1 and MRDC appear to espouse pathways other than cholinergic. Further research for toxicology and exact mode of action is required. 


\section{CONCLUSION}

The hypotensive activity of non-polar extracts and fractions of Moringa oleifera roots and their chemical composition through GC-MS have been established. However, further studies are needed to ascertain its bioactivity, especially in a hypertensive model. This will help to establish the complementary effect of these components and their suitability as an antihypertensive remedy.

\section{REFERENCES}

1. Dhakar RC, Maurya SD, Pooniya BK, Bairwa N, Gupta M, Sanwarmal. Moringa: The herbal gold to combat malnutrition. Chron Y Sci 2011; 2: 119-125.

2. Ross IA. Medicinal plants of world, chemical constituents, traditional and modern uses, 2nd edition. New Jersey 07512: Humana press. Inc.Totowa; 2003. vol 1. $p$. 367-376.

3. Fahey JW. Moringa oleifera: A review of the medical evidence for its nutritional, therapeutic and prophylactic properties. Part 1, TFL J 2005; 1-5.

4. Faizi $S$, Siddiqui BS, Saleem $R$, Noor $F$, Hasnain $S$. Isolation and structure elucidation of a novel glycoside niazidin from the pods of M. oleifera. $J$ Nat Prod 1997; 60: 1317-1321.

5. Nandave M, Ojha SK, Joshi S, Kumari S, Arya DS. Moringa oleifera leaf extract prevents isoproterenolinduced myocardial damage in rats: evidence for an antioxidant, antiperoxidative, and cardioprotective intervention. J Med Food 2009; 12: 47-55.

6. Fakurazi S, Sharifudin SA, Arulselvan P. Moringa oleifera hydroethanolic extracts effectively alleviate acetaminophen-induced hepatotoxicity in experimental rats through their antioxidant nature. Molecules 2012; 17: 8334-8350.

7. Gupta R, Mathur M, Bajaj VK, Katariya P, Yadav S, Kamal R, Gupta RS. Evaluation of antidiabetic and antioxidant activity of Moringa oleifera in experimental diabetes. J Diabetes 2012; 4: 164-171.

8. Dahiru D, Onubiyi JA, Umaru HA. Phytochemical screening and antiulcerogenic effect of Moringa oleifera aqueous leaf extract. Afr $J$ Tradit Complement Altern Med 2006; 3: 70-75.
9. Faizi S, Siddiqui BS, Saleem R, Siddiqui S, Aftab $K$, Gilani AH. Hypotensive constituents from the pods of Moringa oleifera. Planta Med 1998; 64: 225-228.

10. Chuang PH, Lee CW, Chou JY, Murugan M, Shieh BJ, Chen HM. Anti-fungal activity of crude extracts and essential oil of Moringa oleifera Lam. Bioresour Technol 2007; 98 (1): 232-236.

11. Guevara AP, Vargas $C$, Sakurai $H$, Fujiwara $Y$, Hashimoto K, Maoka T, Kozuka M, Ito Y, Tokuda $H$ and Nishino $H$. An antitumor promoter from Moringa oleifera Lam. Mut Res 1999; 440: 181-188.

12. Caceres A, Saravia A, Rizzo S, Zabala L, De Leon E, Nave F. Pharmacologic properties of M.oleifera. 2: Screening for antispasmodic, anti-inflammatory and diuretic activity. J Ethnopharmacol 1992; 36: 233237.

13. Gupta M, Mazumder U k, and Chakrabarti S. CNS activities of methanolic extract of Moringa oleifera root in mice. Fitoterapia 1999; 70: 244-250.

14. Sashidhara KV, Rosaiah JN, Tyagi E, Shukla $R$, Raghubir $R$, Rajendran SM. Rare dipeptide and urea derivatives from roots of Moringa oleifera as potential anti-inflammatory and antinociceptive agents. Eur J Med Chem 2009; 44 (1): 432-436.

15. Farooq A, Sajid L, Muhammad A and Gilani A.H. Moringa oleifera: A Food Plant with multiple medicinal uses. Phytother Res 2007; 21: 17-25.

16. Faizi S, Sumbul S, Versiani MA, Saleem $R$, Sana $A$ and Siddiqui $H$. GC/GCMS analysis of the petroleum ether and dichloromethane extracts of roots of Moringa oleifera, Asian Pac J Trop Biomed 2014; 4(8): 650-654.

17. Marrufo T, Nazzaro F, Mancini E , Fratianni F, Coppola $R$, De Martino L, Agostinho $A B$ and De Feo V. Chemical composition and biological activity of the essential oil from leaves of Moringa oleifera Lam. cultivated in Mozambique. Molecules 2013; 18: 10989-11000.

18. Pino JA. Floral scent composition of Moringa oleifera Lam. J Essent Oil Bear PI 2013; 16 (3): 315-317.

19. Barreto BM, de Freitas JVB, Silveira RE, Bezerra AME, Nunes PE, Gramosa NV. Volatile and non-volatile chemical constituents of M. oleifera Lam, Moringaceae. Rev Bras Farmacogn 2009; 19 (4): 893-897.

20. National Research Council. Guide for the care and use of laboratory animals, 8th edn, The National Academies Press, Washington, DC; 2011. 